\title{
Théâtres et amphithéâtres dans les cités de Gaule romaine : fonctions et répartition
}

\section{Françoise Dumasy}

\section{(2) OpenEdition \\ 1 Journals}

Édition électronique

URL : http://journals.openedition.org/edl/115

DOI : $10.4000 /$ edl. 115

ISSN : 2296-5084

Éditeur

Université de Lausanne

\section{Édition imprimée}

Date de publication : 15 mai 2011

Pagination : 193-222

ISBN : 978-2-940331-25-3

ISSN : 0014-2026

Référence électronique

Françoise Dumasy, «Théâtres et amphithéâtres dans les cités de Gaule romaine : fonctions et répartition », Études de lettres [En ligne], 1-2 | 2011, mis en ligne le 15 mai 2014, consulté le 18 décembre 2020. URL : http://journals.openedition.org/edl/115; DOI : https://doi.org/10.4000/edl.115 


\section{THÉÂTRES ET AMPHITHÉÂTRES DANS LES CITÉS DE GAULE ROMAINE: FONCTIONS ET RÉPARTITION}

On rappellera d'abord la fonction religieuse des théâtres et des amphithéâtres qui se traduit, en Gaule, par des formules spatiales qui mettent en valeur les liens de ces édifices avec les sanctuaires; on explorera en particulier les ressources des compositions sacrées et des compositions urbaines. On insistera ensuite sur leur fonction sociopolitique, la place du calendrier des jeux dans la vie de la cité et le rôle qu'y jouent les magistrats, ainsi que sur la distribution hiérarchisée du public dans la cavea. La carte de localisation de ces édifices dans le cadre des cités soulignera les différences entre Narbonnaise et Trois Gaules invitant à réfléchir aux modalités d'intégration des sanctuaires et des agglomérations dans le réseau religieux des grandes cités de Gaule centrale et septentrionale. On soulignera enfin la fonction ludique de tous les théâtres, même des plus petits d'entre eux.

Depuis une vingtaine d'années, on observe la tendance, chez les auteurs d'ouvrages d'archéologie ou d'architecture antique, à classer théâtres et amphithéâtres parmi les édifices du loisir et à les étudier aux côtés des thermes. On en perçoit bien l'intérêt: il s'agit de privilégier une approche anthropologique qui s'attache aux pratiques antiques mises en parallèle avec les pratiques contemporaines. Et dans le domaine du spectacle, les questionnements sont multiples. Ce faisant, on éloigne quelque peu ces édifices de leurs liens originels avec la cité dans laquelle ils s'élèvent et l'on estompe certaines de leurs fonctions. Nous voudrions revenir ici sur le rôle qu'ils assument dans le cadre des villes ou des sanctuaires en soulignant leur fonction religieuse, mais aussi politique. Et puisqu'on a contesté la fonction ludique de certains théâtres, nous rappellerons les témoignages épigraphiques et archéologiques qui font de tous ces édifices, des lieux de spectacle. 


\section{Religion et théâtre}

On se souvient du texte de Tite-Live sur les origines des ludi scaenici à Rome:

La peste dura cette année-là et la suivante, sous le consulat de Titus Sulpicius Peticus et de Gaius Licinius Stolon [...] [nous sommes en 364 av. notre ère]. L'esprit dominé par la superstition, les Romains organisèrent, dit-on aussi, des jeux scéniques entre autres cérémonies destinées à apaiser le courroux divin. C'était là chose nouvelle chez un peuple guerrier qui n'avait eu auparavant que le spectacle du cirque... ${ }^{1}$

Les jeux scéniques sont institués pour calmer les dieux irrités et l'on fait appel à un rituel étranger, emprunté à l'Etrurie.

Ce lien entre dieux et ludi scaenici reste essentiel tout au long de la période romaine. A la fin du $\mathrm{II}^{\mathrm{e}}$ siècle de notre ère, il est encore souligné - dénoncé en fait - par l'auteur chrétien qu'est Tertullien:

Passons aux représentations théâtrales. Nous avons déjà montré qu'elles étaient solidaires des cavalcades du cirque par une origine commune, des titres semblables (appellation de ludi) et une mise en œuvre connexe: elles en partagent aussi le cérémonial en ce qui regarde la scène. Car on part pour s'y rendre des temples et des autels, de la malédiction du sang et de l'encens, parmi flûtistes et trompettistes (inter tibias et tubas), avec les deux maîtres ignobles des funérailles et des sacrifices, l'ordonnateur et l'haruspice. Par un vice du lieu, le théâtre est en propre un sanctuaire de Vénus... C'est pourquoi le grand Pompée [...] craignit pour sa mémoire quelque blâme des censeurs. Aussi fit-il construire en haut une chapelle de Vénus et dans l'édit appelant le peuple à la dédicace, il ne parla pas d'un théâtre, mais d'un temple de Vénus «au pied duquel, dit-il, nous avons fait mettre des gradins pour les spectateurs $»^{2}$.

Cette atmosphère religieuse qui imprègne la représentation théâtrale et l'édifice lui-même, nous la percevons encore aujourd'hui dans la présence des quelques autels retrouvés dans l'orchestra des théâtres d'Arles, de Vendeuil-Caply ou de Dalheim ou dans celle du temple construit en haut de la cavea à Vienne ou au pied des gradins comme à Vendeuil-

I. Tite-Live, Histoire romaine 7.2.1-8.

2. Tertullien, De Spectaculis 10.1-5. 
Caply $^{3}$. Reconnaissons cependant que cette dernière pratique est rare en Gaule alors qu'elle est bien illustrée dans les théâtres africains ou hispaniques qui ont souvent adopté l'innovation du théâtre de Pompée. En réalité, en Gaule, le patronage divin s'affirme davantage dans des compositions spatiales où le théâtre est construit à proximité immédiate d'un sanctuaire: on y relève des formules variées où sont explorées les ressources du relief, de l'axialité ou de l'alignement pour tisser des correspondances entre sanctuaire et lieu du spectacle. Les modèles en ont été élaborés en Italie et à Rome où le spectacle se donne ante templum in conspectu dei $i^{4}$. Il s'agit donc pour les commanditaires de s'inspirer des réalisations antérieures ou contemporaines et de les adapter au cadre des cités de Gaule romaine.

Camille Jullian est le premier à affirmer que «les théâtres de la Gaule sont tous, sans exception à moi connue, dans le voisinage et la dépendance d'un temple. Tous font partie d'un terrain ou d'un enclos sacré» 5 . Quelques décennies plus tard, Paul-Marie Duval insiste sur la présence fréquente d'un temple dans l'axe du théâtre en s'appuyant sur les exemples souvent cités d'Avenches, d'Augst et de Mandeure ${ }^{6}$. Aujourd'hui, on saisit mieux les théâtres dans leur environnement architectural ou urbanistique grâce au développement des prospections aériennes et géophysiques et à de nombreuses interventions archéologiques. Il ne s'agit pas ici d'en livrer une étude exhaustive, mais d'en évoquer les traits essentiels. L'analyse permet de distinguer deux séries, l'une que nous qualifierions de composition sacrée, bien représentée dans les grands sanctuaires urbains, suburbains ou dans ceux qui sont dispersés sur le territoire des cités: le théâtre y tient une place remarquable dans la conception d'ensemble. Nous désignerions la seconde par l'expression de composition urbaine dans la mesure où l'emplacement du théâtre s'apprécie à l'échelle de la ville par une analyse subtile de son tissu et de sa trame.

3. Arles: P. Gros, La Gaule Narbonnaise, p. 46-48; Vendeuil-Caply: G. Dufour, "Le grand théâtre de Vendeuil-Caply (Oise)»; Dalheim: J. Krier, "Le théâtre galloromain découvert en 1985 à Dalheim (Grand Duché de Luxembourg) ", p. 121-123; Vienne: J. Formigé, Le théâtre romain de Vienne.

4. Il s'agit d'une précision donnée à propos des Ludi Megalenses: cf. J. A. Hanson, Roman theater-temples, p. 9-26.

5. C. Jullian, "Les arènes de Lutèce et les théâtres amphibies de la Gaule», p. 189.

6. P.-M. Duval, "Théâtres et édifices sacrés», p. 360. 


\subsection{Compositions sacrées}

La première catégorie est bien illustrée par le sanctuaire de la Fontaine à Nîmes. L'architecte a implanté le théâtre dans l'alignement d'un des portiques du sanctuaire identifié par Pierre Gros comme un Augusteum ${ }^{7}$. Ici le lieu de culte préromain, installé autour d'une source et dédié au dieu topique Nemausus, a été transformé en lieu de célébration du culte impérial dès 25 av. J.-C. Le théâtre parachève le processus de captation en accueillant des ludi scaenici, et probablement au II siècle de notre ère, comme le suggèrent des inscriptions retrouvées près de l'autel, le programme musical des concours à la grecque ${ }^{8}$. On ignore cependant si l'édifice fait partie de la composition d'origine ou s'il est construit dans un second temps. On peut seulement constater ses dimensions modestes - son diamètre de $40 \mathrm{~m}$ ne lui permet d'accueillir que trois à quatre mille spectateurs - et sa situation latérale: il ne donne pas sur l'un des trois portiques disposés en pi qui délimitent la zone sacrée proprement dite. Malgré nos lacunes dans la connaissance du dispositif d'ensemble, on identifie ici l'une des formules les plus précoces de l'intégration du théâtre à un sanctuaire gallo-romain (si du moins le théâtre est bien contemporain du réaménagement augustéen).

Pendant longtemps, on a pensé que le théâtre, également augustéen, de Lyon illustrait une autre formule de composition sacrée: adossé à la colline de Fourvière et construit au pied du sanctuaire dit de Cybèle, il renvoyait à la position fréquente du théâtre dominé par un temple, l'une des formules les plus abouties du complexe architectural du theatertemple étudié par John Arthur Hanson 9. Or les fouilles réalisées par Armand Desbat ont montré que le sanctuaire de Cybèle n'avait jamais existé, ce qui impose une nouvelle lecture de ce secteur ${ }^{10}$. En réalité, le théâtre a été construit en bordure de la première trame coloniale; il a été installé sur le flanc sud-est du plateau de La Sarra pour profiter d'une

7. P. Gros, «L'Augusteum de Nîmes».

8. Les recherches de Maria Letizia Caldelli ont bien mis en évidence l'instauration de ces concours qui se tenaient non seulement à Nîmes, mais aussi à Marseille, à Vienne et peut-être même à Lyon. Cf. M. L. Caldelli, «Gli agoni alla greca nelle regioni occidentali dell'Impero".

9. J. A. Hanson, Roman theater-temples.

Io. A. Desbat, «Nouvelles recherches à l'emplacement du prétendu sanctuaire lyonnais de Cybèle», p. 263-266. 
pente naturelle ${ }^{11}$. Est-il pour autant isolé? On observe qu'en arrière de la frons scaenae se déploie selon les mêmes orientations une esplanade entourée de trois portiques doubles dessinant un plan en pi, tandis que le quatrième côté, qui fait face au théâtre, est fermé par un simple mur d'enceinte. Cette esplanade, identifiée initialement comme le forum de Plancus et toujours très mal connue, pourrait être en réalité une porticus post scaenam et l'on pourrait même envisager, comme à Mérida ${ }^{12}$, la présence d'un temple sur le quatrième côté. On aurait alors affaire à la formule du face à face qui place le théâtre sur le même axe que le temple, soulignant ainsi la place que tiennent les ludi scaenici dans la célébration du culte. Mais en l'absence de données archéologiques, il ne s'agit que d'une hypothèse.

En revanche, les fouilles menées récemment à Augusta Raurica, autre colonie fondée par Plancus, révèlent que dans les années 70-80 de notre ère, un théâtre en pierre est construit sur la retombée du plateau occupé par la colonie, face à la colline sacrée du Schönbühl ${ }^{13}$. L'édifice s'insère dans une composition monumentale rigoureuse puisqu'au même moment, est élevé un grand temple de type romain qui occupe la majeure partie de l'ancien sanctuaire dont subsistent quelques constructions. Temple et théâtre partagent désormais le même axe et se dressent à la même hauteur puisque les gradins de la cavea répondent à l'escalier monumental qui permet d'accéder au péribole du temple. A la fin du $\mathrm{II}^{\mathrm{e}}$ siècle, lors de la reconstruction du théâtre, après sa transformation en amphithéâtre, il semble bien que l'on ne construise pas de frons scaenae et qu'on laisse ouverte la perspective entre la cavea et la cella. Exemple remarquable d'une association qui va jusqu'à supprimer un élément essentiel à la représentation pour lui substituer la façade du temple luimême.

A la fin du I $^{\text {er }}$ siècle, cette composition est reprise dans la colonie d'Avenches: un vaste espace à l'origine marécageux, situé en bordure de la zone urbanisée mais à l'intérieur de l'enceinte, accueille un ensemble monumental très structuré où théâtre et temple se font face de part et

II. A. Desbat, "La colonie de Plancus», p. 65 sq.

I2. W. Trillmich, "Un sacrarium del culto imperial en el teatro de Merida».

I3. P.-A. Schwarz, "Zur Chronologie und Typologie der drei Theaterbauten von Augusta Rauricorum». 
d'autre d'une longue esplanade ${ }^{14}$. Les échanges entre les deux édifices y sont favorisés par l'ouverture d'entrées frontales de part et d'autre de la scène et dans les aditus. Cette perméabilité entre les deux édifices a été facilitée par l'adoption, dans un grand théâtre d'inspiration romaine, d'une scène de type gallo-romain qui s'avance dans l'orchestra et libère le mur de fermeture. Ce tête à tête est modifié dans la seconde moitié du $\mathrm{II}^{\mathrm{e}}$ siècle par la construction de deux fana qui font évoluer le dispositif initial en introduisant de nouveaux cultes, probablement des divinités du territoire de la cité. Il faut ajouter que cet ensemble s'insère dans une aire plus vaste, traversée par une voie qui mène à la Porte de l'Ouest, aire qui accueille l'amphithéâtre et plusieurs sanctuaires. Il y a là un vaste quartier monumental et religieux qui se développe à intérieur de l'enceinte, mais en dehors de la zone quadrillée réservée à l'habitat et au forum.

Malgré son efficacité, ce dispositif ne paraît guère avoir été adopté dans une autre ville de Gaule. Seule une agglomération séquane, Mandeure, s'en inspire pour construire en bordure de la zone urbanisée un vaste ensemble monumental ${ }^{15}$. Comme à Avenches, le face à face théâtre-temple est enrichi par la présence, aux côtés de l'édifice axial, d'autres temples, mais on ignore la date d'apparition des différents éléments. On peut s'étonner que cette composition, qui met le théâtre sur le même rang que le temple, n'ait pas connu une plus large diffusion. Elle semble cantonnée à trois cités voisines: il pourrait s'agir de l'innovation d'un architecte qui n'aurait pas été adoptée hors du cadre régional.

Et cependant, la ville de Trèves possède également un très vaste sanctuaire établi à la sortie de la vallée de l'Alt, en bordure du tissu urbain. Il sera inclus dans le périmètre urbain lors de la construction de l'enceinte à la fin du II siècle. On y a mis au jour plusieurs dizaines de fana dont les plus anciens remontent à l'époque augustéenne. Dans les années 100 est édifié un théâtre de type gallo-romain de quelque $55 \mathrm{~m}$ de diamètre: lui fait face, à $30 \mathrm{~m}$ en arrière de la scène, un fanum construit sur le même axe que lui. On retrouve le principe du face à face, mais il est appliqué ici à un ensemble modeste qui n'est qu'une composante d'un sanctuaire de cinq hectares. D’une capacité d'accueil limitée, peut-être réservé en priorité aux fidèles des dieux honorés dans le fanum - sans qu'il s'agisse pour

I4. G. Matter, Das römische Theater von Avenches/Aventicum, p. 178-183.

I5. E. Frézouls, Les villes antiques de la France; cf. aussi M. Thivet, Méthodes nouvelles de l'archéologie appliquées au site antique de Mandeure/Mathay (Doubs). 
autant d'un théâtre cultuel, comme cela a été souvent affirmé - le théâtre est détruit dans les années $200^{16}$.

Mais Trèves possède un autre théâtre, associé au sanctuaire suburbain de Lenus Mars. On n'en connaît que quelques murs rectilignes qui suggèrent un diamètre d'une centaine de mètres. Or, la composition choisie ici - ou imposée par le terrain - est celle de l'implantation côte à côte et, pour le théâtre, de l'adossement à la colline. Les travaux récents ont montré qu'il s'agissait d'un sanctuaire public érigé en l'honneur du grand dieu trévire de l'époque romaine, Lenus Mars ${ }^{17}$. Avec la présence de deux théâtres dans des contextes religieux différents, Trèves illustre l'importance d'un tel édifice dans le déroulement des rituels. Apparaît également la spécificité de ces sanctuaires suburbains dont on ne connaît en Gaule pour l'instant que quelques exemples. A Autun ${ }^{18}$ et à Meaux ${ }^{19}$, ils dotent la ville d'un second théâtre. A Clermont-Ferrand ${ }^{20}$ comme dans l'agglomération biturige d'Argentomagus ${ }^{21}$, ils captent à leur bénéfice le seul théâtre qui existe. Les théâtres qui ont pu être fouillés révèlent des dispositifs de type gallo-romain, avec en particulier la scène dans l'orchestra et les grandes entrées frontales face à la cavea. Quant aux temples monumentalisés, ils ont souvent connu une première phase comme fana. Leur situation en périphérie du chef-lieu ou de la colonie révèle probablement des liens étroits avec le reste du territoire: ils pourraient renvoyer à d'anciens dieux, devenus protecteurs de la cité et désormais romanisés, comme on le voit au sanctuaire de Lenus Mars.

On comprendrait mieux alors les choix faits par certains évergètes d'adopter dans ces ensembles suburbains les caractéristiques architecturales des édifices élevés dans les grands sanctuaires de la cité. La partie proprement sacrée y occupe souvent une vaste superficie: s'y dressent un ou plusieurs temples agrandis et embellis à diverses reprises. Lorsque le sanctuaire est installé sur une hauteur, le théâtre est construit en

16. E. Gose, Der gallo-römische Tempelbezirk im Altbachtal zu Trier.

I7. T. Derks, "Le grand sanctuaire de Lenus Mars à Trèves et ses dédicaces privées", p. 241-243.

I8. A. Rebourg, «L'urbanisme d'Augustodunum (Autun, Saône-et-Loire)», p. 158-160 et 218 .

19. D. Magnan, "Les trois édifices de spectacle antiques de Meaux (Seine-etMarne)».

20. Ch. Le Barrier, Ceyrat (Puy de Dôme), Puy de Montaudou.

2I. F. Dumasy, Le théâtre d'Argentomagus (Saint-Marcel, Indre), p. 48-52. 
contrebas, étageant ses gradins sur la pente et déroulant ses jeux sous le regard des dieux. C'est le cas à Saint-Cybardeaux ${ }^{22}$ ou à Tintignac (Naves, Corrèze) où la fouille a confirmé, en les précisant, les situations des différents temples et en particulier l'extraordinaire dispositif semicirculaire qui domine le théâtre ${ }^{23}$. Lorsque le terrain est plat, on installe souvent le mur rectiligne du théâtre dans l'alignement de l'un des murs du péribole. A Genainville, ${ }^{24}$ à Blicquy ${ }^{25}$, à Clion-sur-Indre ${ }^{26}$, à Vendeuvre-du-Poitou ${ }^{27}$, la position latérale du théâtre rappelle celle de l'édifice de Nîmes. A Ribemont-sur-Ancre, le théâtre est construit sur l'axe de composition de l'ensemble, non pas face mais dos au sanctuaire qui se dresse à $300 \mathrm{~m}$. Ici, les espaces investis par l'ensemble d'époque romaine se superposent aux grandes esplanades laténiennes ${ }^{28}$.

Les théâtres présentent les caractéristiques essentielles de la série galloromaine ${ }^{29}$ - notamment le plateau scénique installé dans l'orchestra, le postscaenium adossé au mur de scène et les entrées d'honneur ouvertes dans le mur rectiligne - déclinées dans toutes ses variantes ${ }^{30}$. Leur répondent toujours des sanctuaires à fana, même si dans leur dernière version, les temples présentent souvent des plans et des décors qui les rapprochent de leurs homologues de tradition romaine. Il faut donc se demander ce qui a justifié la localisation de ces sanctuaires et en particulier s'ils succèdent à un lieu de culte de l'Age du fer. Les réponses ne concernent qu'un nombre restreint de sites. A Meaux, à Ribemontsur-Ancre ou à Tintignac, le sanctuaire gaulois est attesté, ailleurs il n'est souvent que supposé. La recherche future aura à préciser ces hypothèses, mais il est possible que certains de ces sanctuaires ne surgissent qu'à l'époque augustéenne, voire plus tard et ne révèlent en fait aucun

22. Ch. Vernou, La Charente, p. 276 sq.

23. Ch. Maniquet, Le sanctuaire antique des Arènes de Tintignac, p. 69-82.

24. D. Vermeersch, M. Wabont, "Genainville», p. 272.

25. E. Gillet, N. Paridaens, L. Demarez, "Le sanctuaire de Blicquy-"Ville d'Anderlecht" (prov. Hainaut, Belgique)», p. 211-215.

26. J. Holmgren, "Un sanctuaire gallo-romain avec théâtre à Clion-sur-Indre», p. 17. 27. M.-R. Aucher, M. Aucher, "Le théâtre gallo-romain des Tours Mirandes à Vendeuvre-du-Poitou ".

28. J.-L. Brunaux, «Ribemont-sur-Ancre (Somme)», p. 133 sq. et 154 sq.

29. Nous reprenons ici l'expression créée par K. Kenyon lors de la publication du théâtre de Verulamium; cf. K. Kenyon, "The Roman theater at Verulamium».

30. F. Dumasy, "Les édifices de spectacle en Gaule du Nord», p. 450-459. 
antécédent gaulois ${ }^{31}$. Dans tous les cas, c'est aux décisions des élites des cités que ces sites doivent leur existence. Rien d'étonnant dans ces conditions que, tout en répondant aux exigences et aux règles romaines, temples et théâtres révèlent des traits spécifiques à leur implantation régionale et qu'ils soient les uns et les autres de type gallo-romain.

\subsection{Compositions urbaines}

Elles s'appliquent en priorité à la ville, mais elles peuvent aussi concerner les agglomérations de la cité, dans la mesure où celles-ci développent un tissu urbain. Les colonies d'Arles et d'Orange sont parmi les premières à intégrer le théâtre dans le quadrillage, tout en exploitant sa portée religieuse. Le théâtre d'Arles, édifié dans les années 20-10 av. notre ère en bordure de la zone urbanisée ${ }^{32}$, est parfaitement aligné sur les axes et fait face au forum; le décalage de leur implantation y est compensé par une liaison directe assurée par le decumanus principal. A Orange, théâtre et sanctuaire, adossés à la colline et construits côte à côte selon la même orientation, dominent de leur masse les insulae qui se déroulent à leurs pieds. Consacré au culte impérial et réalisé dès l'époque julioclaudienne, cet ensemble illustre la place que tient le théâtre dans les célébrations impériales et les ressources qu'il offre à des compositions urbaines fortes ${ }^{33}$. Pour Orange, Pierre Gros invoque l'exemple réalisé plus tardivement de Brescia, tandis que pour Arles, il renvoie à la composition parfaitement intégrée d'Augusta Bagiennorum. Or ces réussites narbonnaises, proches de réalisations italiennes, ne trouvent guère d'écho en Gaule: citons Saint-Bertrand-de-Comminges où le théâtre, adossé à la colline qui domine la ville et implanté selon les mêmes axes que les autres monuments publics, intervient comme un élément de la composition urbaine ${ }^{34}$; citons aussi le théâtre de Vienne, dominé par le

3I. Comme au sanctuaire de la forêt d'Halatte, cf. M. Durand, "Un sanctuaire "rural" ", p. 128.

32. A. Roth-Congès, "L'acanthe dans le décor architectonique proto-augustéen en Provence».

33. P. Gros, La Gaule Narbonnaise, p. 50-54.

34. J.-L. Paillet, C. Petit, «Nouvelles données sur l'urbanisme de Lugdunum des Convènes". 
sanctuaire du mont Pipet, mais ici la topographie tourmentée de la ville ne permet pas les implantations axiales ${ }^{35}$.

Un certain nombre de villes font le choix d'intégrer le théâtre à la trame urbaine en jouant sur un emplacement suggestif. A Limoges et à Jublains, le théâtre est construit à l'extrémité de la rangée centrale des insulae qui constitue l'axe monumental de la ville ${ }^{36}$. A Alesia, dès le règne de Claude, on lance la construction d'un premier théâtre en l'adossant à l'extrémité du plateau qui porte le sanctuaire et les secteurs d'habitats: la courbe de la cavea tourne le dos au temple, mais, encadré par deux rues et occupant la largeur de deux insulae, le théâtre constitue un remarquable signal monumental ${ }^{37}$.

A Fréjus, Apt ou Lutèce en revanche, l'insula choisie n'offre rien de remarquable ${ }^{38}$, mais la liaison rapide avec le centre monumental y est assurée par sa place dans le réseau viaire. Lorsque le théâtre ne peut constituer avec le temple un ensemble intégré, on tente de le construire non loin d'un sanctuaire et on exploite les ressources de la proximité: à Beaumont-sur-Oise, un fanum se trouve dans l'insula voisine du théâtre ${ }^{39}$; à Dalheim, le théâtre est construit au pied du plateau, à l'écart des insulae, mais à quelques centaines de mètres d'une vaste aire cultuelle ${ }^{40}$. Il est clair que l'emplacement du théâtre dans le cadre urbain varie en fonction de nombreux facteurs - relief, date de construction, terrains disponibles, etc. Mais, dans tous les cas, le théâtre est inséré dans un circuit religieux tracé lors des processions qui relient les différents secteurs de la ville et rassemblent la population.

35. A. Le Bot-Helly, "Vienne du village gaulois à la capitale de cité ( $5^{\mathrm{e}}$ siècle av. J.-C./2e siècle apr. J.-C.)», p. 108.

36. J.-P. Loustaud, Limoges antique; J. Naveau, «Urbanisme et occupation du sol à Jublains», p. 78.

37. F. Rossi, Théâtre d'Alesia.

38. Elle offrait probablement des avantages topographiques (la présence d'un relief) ou urbanistiques (un terrain libre). L. Rivet, Atlas topographique des villes de Gaule méridionale; P. De Michele "Découvertes récentes sur le théâtre antique d'Apt»; D. Busson, "Lutèce monumentale du Ir au IVe s.", p. 261-263.

39. D. Vermeersch, "L'agglomération antique de Beaumont-sur-Oise (Val d'Oise)», p. 118.

40. J. Krier, "Le théâtre gallo-romain découvert en 1985 à Dalheim (Grand Duché de Luxembourg)», p. 125. 


\section{Religion et amphithéâtre}

A cause de leur plan ovalaire, de leurs dimensions et de leur construction postérieure au développement urbain initial, la plupart des amphithéâtres sont élevés à la périphérie de la ville, à l'extérieur du plan quadrillé. Loin d'être isolés, ils sont souvent construits à proximité d'une voie importante sur laquelle ils orientent leurs grandes entrées. Ainsi à Bordeaux, à Limoges, à Metz ou à Senlis, l'amphithéâtre apparaît comme un signal monumental qui, placé à l'entrée ou à la sortie de la ville, en proclame la richesse et la romanité ${ }^{41}$. S’y greffe-t-il une signification religieuse liée à l'édition des munera?

Comme le théâtre, et peut-être de façon plus systématique que lui, l'amphithéâtre comporte à l'intérieur de ses murs un temple. Il s'agit du sacellum installé au bas de la cavea. Son identification n'est pas toujours facile, car son emplacement varie d'un édifice à l'autre: à Lyon, à Fréjus, à Cimiez, à Avenches, il se situe à l'une des extrémités du petit axe et un escalier le relie à la loge d'honneur ${ }^{42}$. Dans d'autres cas, la chapelle n'a de communication qu'avec l'arène et ne se distingue guère des carceres. La présence de niches pour les statues cultuelles ou la découverte d'un autel sont alors déterminantes pour son identification. On observe que certains dieux entretiennent des relations privilégiées avec l'arène: Hercule à qui est dédiée une chapelle à l'amphithéâtre de Senlis, Mars, seul ou en association avec Diane ${ }^{43}$. A partir des $\mathrm{II}^{\mathrm{e}}$ et $\mathrm{III}^{\mathrm{e}}$ siècles, c'est Némésis qui est surtout invoquée. Elle apparaît comme la déesse directement liée aux combats de l'arène. Faut-il conclure de la présence de ce lieu de culte que l'amphithéâtre possède une certaine autonomie religieuse et que la liaison avec un temple extérieur ne s'impose pas? Il semble en fait que le sacellum était destiné essentiellement aux gladiateurs qui y faisaient leurs dévotions lors de la pompa et qu'il ne tenait nullement lieu de temple public. Ce lieu de culte interne n'exclut donc nullement que l'amphithéâtre puisse être associé à un sanctuaire urbain.

La meilleure illustration en est le choix fait en 19 de notre ère par le notable santon Caius Iulius Rufus de faire construire un amphithéâtre

4I. F. Dumasy, «Les édifices de spectacle», p. 301.

42. J.-C. Golvin, L'amphithéâtre romain, p. 337-340.

43. A l'amphithéâtre d'Arles, Diane chasseresse était représentée dans une niche de la galerie du rez-de-chaussée. 
à l'autel fédéral des Trois Gaules. L'édifice y apparaît comme un lieu de célébration du culte impérial ${ }^{44}$. C'est un choix identique qui est fait au sanctuaire provincial de Narbonne où l'amphithéâtre est associé au temple dans une composition qui joue sur l'alignement des axes et l'équilibre des masses ${ }^{45}$. Peut-être faut-il rapprocher de la formule lyonnaise le choix des notables d'Amiens d'implanter l'amphithéâtre à l'extrémité du forum. Exemple unique en Gaule, et ailleurs, d'un amphithéâtre bâti en plein cœur d'une ville et selon les orientations mêmes du forum ${ }^{46}$. Rappelons également qu'entre 100 et 200, l'amphithéâtre d'Augst vient prendre la place du théâtre dans le face à face avec les divinités.

A Autun, l'amphithéâtre est édifié à l'intérieur de l'enceinte, aux côtés du théâtre avec lequel il compose un quartier des spectacles comme à Aoste ou à Mérida. Or, il apparaît comme l'édifice privilégié du couple puisque son petit axe est situé dans le prolongement d'une rue qui le relie directement au forum, alors que le théâtre est en situation latérale ${ }^{47}$. A Trèves, l'amphithéâtre est construit dans l'alignement du decumanus maximus et à l'extrémité de l'axe monumental qui jalonne le développement de la ville depuis le pont sur la Moselle ${ }^{48}$. A Avenches, nous l'avons vu, l'amphithéâtre appartient à un vaste secteur religieux lié au spectacle.

Ces diverses formules - insertion dans un sanctuaire, alignement sur les axes du forum, liaison rapide avec le réseau viaire - sont autant de signes de la place que prend progressivement l'amphithéâtre dans le calendrier religieux de la cité. Si le théâtre est l'édifice de spectacle majeur dans les compositions julio-claudiennes des villes où il assure les célébrations du culte impérial, on observe quaprès la construction de l'amphithéâtre flavien à Rome, nombre de cités se dotent de l'édifice réservé aux munera et aux venationes.

44. P. Wuilleumier, Inscriptions latines des Trois Gaules, $\mathrm{n}^{\circ} 217$.

45. M. Gayraud, Narbonne antique des origines à la fin du IIIe siècle.

46. D. Bayard, J.-L. Massy, Amiens romain, Samarobriva Ambianorum, p. 86-94.

47. Pour justifier cette position secondaire du théâtre, Alain Rebourg évoque son adossement à un léger relief, mais on aurait pu choisir d'en faire profiter l'amphithéâtre. Ce n'est pas la décision qui a été prise: priorité a été donnée à l'axialité amphithéâtreforum. Cf. A. Rebourg, "L'urbanisme d'Augustodunum (Autun, Saône-et-Loire)", p. 188-193.

48. P.-H. Kuhnen, «Trèves-Augusta Treverorum». 


\section{Fonction socio-politique}

Nous rappellerons ici quelques aspects de la participation des édifices de spectacle non seulement au paysage urbain et religieux de la cité, mais aussi aux rituels par lesquels la communauté affirme et renforce sa cohésion.

\subsection{Le calendrier des jeux}

Faute d'inscription, il est difficile d'en reconstituer le rythme et les exigences dans les cités de Gaule. Du moins pouvons-nous relire dans la constitution municipale de la colonie d'Urso en Bétique, les chapitres qui réglementent l'editio régulière des spectacles publics par les magistrats municipaux, notamment les chapitres LXX et LXXI consacrés aux ludi scaenici et aux munera ${ }^{49}$ :

Ilviri... in suo mag(istratu) munus ludosve scaenicos Iovi, Iunoni Minervae deis deabusq(ue) quadriduom m(ajore) p(arte) diei... Aediles... munus ludos scaenicos Iovi Iunoni Minervae triduom... et unum diem in circo aut in foro Veneri

Les duoviri ont à organiser quatre jours de munus ou de jeux scéniques dédiés à la triade capitoline et à l'ensemble des dieux et déesses - le choix du spectacle leur est laissé - tandis qu'on attend des édiles trois jours de représentations auxquels s'ajoute une journée donnée au cirque ou au forum, consacrée à Vénus. On peut supposer que chaque année, dans les colonies de Gaule romaine, les magistrats remplissaient des obligations du même type. Cependant comme la loi d'Urso date de 44 av. notre ère, ces prescriptions ont connu des évolutions. A partir du règne d'Auguste, le culte impérial s'impose, captant à son bénéfice les diverses manifestations religieuses et incitant à la diffusion des spectacles. Les quelques inscriptions commémoratives de la construction de théâtres ou d'amphithéâtres dans les Trois Gaules suggèrent que les magistrats des chefslieux assument les mêmes charges que ceux des colonies ${ }^{50}$. Cependant

49. Lex coloniae Iuliae Genetivae (Urso en Bétique), (CIL II 5439).

50. Pour l'Aquitaine, voir F. Dumasy, «Les édifices de spectacle», p. 304 sq.; pour la Lyonnaise, voir l'inscription de Feurs, CIL XIII 1642; pour la Belgique, voir l'inscription d'Eu, cf. M. Dondin-Payre, "Sanctuaires publics et territoires civiques». 
nous verrons plus loin que la carte de répartition des édifices de spectacle en Gaule invite à s'interroger sur les conditions dans lesquelles les règles étaient appliquées.

\subsection{L'accès du public et sa répartition dans la cavea}

Il s'agit d'évoquer les dispositifs qui contribuent à faire des édifices de spectacle des lieux où s'affirme la hiérarchie sociale et où se révèle la position de chacun au sein du groupe. Chaque édifice est en effet confronté aux problèmes de la circulation du public et de sa répartition dans la cavea. Nous n'envisagerons pas ici les solutions proposées dans les édifices à structure creuse - théâtres ou amphithéâtres du chef-lieu -, car ils bénéficient d'un système complexe et efficace de couloirs et d'escaliers installés dans les substructures de la cavea. Nous voudrions plutôt observer la façon dont les édifices de spectacle, construits en dehors du chef-lieu, avec des moyens limités, répondent aux obligations du cahier des charges. Le respect de la hiérarchie sociale impose que les différentes classes disposent de circuits spécifiques qui évitent toute rencontre. Comment procéder lorsque l'on ne dispose pas des ressources de la circulation interne?

La caractéristique essentielle de la série gallo-romaine tient à la réduction de la longue scène rectangulaire en un plateau, de même profondeur mais de longueur réduite, qui s'installe dans l'orchestra et libère des espaces latéraux. Désormais une porte est percée, de chaque côté de la scène, dans le mur périmétral rectiligne. Cette entrée frontale est réservée aux notables qui pénètrent directement dans l'orchestra sous le regard $\mathrm{du}$ public et gagnent leur siège. Bénéficient des mêmes accès directs les occupants des premiers rangs de la cavea qui, au théâtre d'Argentomagus, utilisent un escalier de quelques marches pour franchir le mur qui sépare l'orchestra de la cavea et monter dans les premiers gradins ${ }^{51}$. La suppression des grandes entrées simplifie le schéma de circulation, puisque les spectateurs privilégiés sont conduits vers le mur périmétral rectiligne, tandis que le reste du public se répartit autour du mur périmétral curviligne.

L'accès par les parties hautes est la règle toujours suivie. Si le mur curviligne ne comporte pas d'ouverture, ce qui est possible dans les

5I. F. Dumasy, Le théâtre d'Argentomagus (Saint-Marcel, Indre), p. 228-230. 
premières phases des édifices construits sur remblai, des escaliers adossés permettent aux spectateurs d'atteindre le sommet de la cavea pour descendre ensuite dans les gradins. Plus souvent s'ouvrent dans ce mur des portes qui donnent accès à des passages rayonnants, allées à ciel ouvert ou couloirs voûtés. Il est fréquent que des escaliers s'ouvrent dès la porte franchie pour desservir les gradins les plus élevés. Quant aux passages rayonnants, ils emmènent les spectateurs vers une première allée concentrique qui les répartit dans la cavea médiane. Souvent des escaliers installés en haut, au milieu ou au bas de ces allées permettent une distribution rapide du public et compense l'absence de toute circulation interne. Il est évident que chaque édifice, tout en s'inspirant de ces principes, invente son propre plan de circulation. Mais faute de fouilles exhaustives, on ne dispose que d'un échantillon réduit d'informations.

\subsection{Le gradin d'honneur}

A l'amphithéâtre, les places d'honneur sont installées sur la plateforme du podium qui peut en offrir plusieurs rangs. Au théâtre, l'orchestra n'étant plus utilisée pour les évolutions du chœur, cet espace semicirculaire est désormais réservé aux gradins d'honneur: un, deux, trois emmarchements larges et peu élevés accueillent de confortables sièges à dossier, réalisés souvent en marbre. Dans les chefs-lieux, ces places sont réservées aux décurions, tandis que les premiers gradins de l'ima cavea le sont aux corps constitués parmi lesquels figurent notamment les sévirs augustaux ${ }^{52}$. On a longtemps considéré que les théâtres de type galloromain n'étaient pas concernés par ce dispositif, puisque les agglomérations ou les sanctuaires dans lesquels ils s'élèvent ne disposent d'aucun pouvoir politique et ne bénéficient d'aucun corps officiel.

Or, la fouille du second théâtre d'Argentomagus a révélé, en 1987, l'existence d'une structure adossée au mur qui assure la séparation entre l'orchestra surcreusée et les premiers gradins de la cavea appuyés au relief naturel. Composée d'une maçonnerie large de $0,60 \mathrm{~m}$, puis $0,20 \mathrm{~m}$ plus bas d'une autre, large de $0,40 \mathrm{~m}$, devant laquelle court une bande de sable de $1 \mathrm{~m}$ de large limitée par un rang de moellons, elle suivait la courbe outrepassée du mur sur une longueur de $65 \mathrm{~m}$. Des traces de

52. J. Kolendo, «La répartition des places aux spectacles et la stratification sociale dans l'Empire romain». 
mortier de tuileau sur la maçonnerie adossée indiquaient qu'elle était recouverte d'un placage ${ }^{53}$. Il s'agissait manifestement d'un gradin, plus large que les autres, accompagné de son marchepied et précédé d'une allée de circulation, un gradin d'honneur réservé aux personnalités.

Contrairement à ce que dit Monique Dondin-Payre, un tel dispositif n’a jamais été regardé comme une "innovation" ${ }^{54}$. Réalisé dans les années 180 de notre ère, il serait intervenu bien tard dans l'histoire du théâtre en Gaule puisque c'est sous le règne de Claude que l'on voit apparaître les premiers théâtres dans les agglomérations de la cité. En réalité, sa découverte a constitué une révélation archéologique, car il n'avait jamais été identifié ailleurs. Au-delà de la remise en cause de la théorie du théâtre-amphithéâtre - Albert Grenier voyait dans le mur séparant la cavea de l'orchestra la fondation du podium ${ }^{55}$ - il fallait nous assurer qu'il ne s'agissait pas d'un cas isolé et nous interroger sur sa date d'apparition. Comme l'orchestra du premier théâtre d'Argentomagus avait disparu lors de la reconstruction du second, nous avons mené notre enquête sur les autres sites pour retenir, outre quelques cas hypothétiques, l'exemple du théâtre de Saint-Cybardeaux où le second état - fin $\mathrm{II}^{\mathrm{e}}$ ou début $\mathrm{III}^{\mathrm{e}}$ siècle - présente dans l'orchestra trois emmarchements semi-circulaires qui réutilisent en partie des gradins inscrits de l'état antérieur ${ }^{56}$. Depuis, les théâtres d'Epiais-Rhus et de Dalheim ont révélé plusieurs rangs de sièges d'honneur avec dossier et nous savons qu'à Dalheim, une première rangée est mise en place dès la construction du théâtre dans la seconde moitié du I ${ }^{\mathrm{er}}$ siècle ${ }^{57}$. Nous pouvons donc affirmer que la présence d'un gradin d'honneur est une pratique habituelle dans les théâtres des agglomérations et des sanctuaires de la cité. Il reste maintenant à s'interroger sur la personnalité des bénéficiaires.

Lors de la publication du théâtre d'Argentomagus, nous avions évoqué la possibilité que "les décurions du chef-lieu viennent assister aux

53. F. Dumasy, Le théâtre d'Argentomagus (Saint-Marcel, Indre), p. 195-197 et 230 sq.

54. M. Dondin-Payre, «Sanctuaires publics et territoires civiques», p. 153.

55. Nous ne développerons pas ici ce point qui relève de l'approche historiographique des théâtres de type gallo-romain car il exigerait une longue exposition; cf. A. Grenier, Manuel d'archéologie gallo-romaine, p. 880-974.

56. C. de La Croix, Etude sur le théâtre gallo-romain des Bouchauds.

57. M. Wabont, F. Abert, D. Vermeersch, Le Val-d'Oise, p. 236 ; J. Krier, «Le théâtre gallo-romain découvert en 1985 à Dalheim (Grand Duché de Luxembourg)», p. 123. 
spectacles du théâtre dans des villes dont ils sont parfois les patroni» ${ }^{58}$. Nous ajoutions que "l'absence de théâtre au chef-lieu ne leur laissait d'ailleurs pas d'autre possibilité s'ils voulaient offrir des ludi scaenici» ${ }^{59}$. Le nombre des places d'honneur - plus d'une soixantaine à Argentomagus - nous semblait justifier cette vision des choses et nous rappelions les inscriptions de Vendoeuvres-en-Brenne et de Néris-les-Bains signalant l'intervention de notables du chef-lieu dans la construction des édifices publics des agglomérations ${ }^{60}$. Cependant, il paraissait difficile, pour la plupart des spécialistes, de déduire des seuls témoignages architecturaux et non de données épigraphiques, le déplacement des décurions vers les agglomérations et leur assistance aux ludi scaenici qui y sont donnés: leur réserve nous incitait à la prudence. Mais Monique DondinPayre vient de confirmer que "ceux qui viennent dans les théâtres des agglomérations et y sont distingués sont ceux qui administrent la cité puisque les relations entre chef-lieu et agglomérations ne sont pas définies par le cloisonnement mais par la complémentarité " ${ }^{61}$. La présence d'un gradin d'honneur dans ces théâtres apparaît donc comme un signe de plus des liens qui existent entre chef-lieu et agglomérations.

\subsection{La répartition des édifices de spectacle dans les cités de Gaule}

Publiée lors du colloque de Lattes en 1989, la carte des édifices a été reprise et complétée ${ }^{62}$ (fig. 1). Sa réalisation obéit à un double objectif: localiser tous les théâtres et tous les amphithéâtres - les cirques et les odéons en sont pour le moment exclus - dans le cadre des cités et des provinces; caractériser les édifices et cartographier leur classement typologique. Il a fallu isoler les édifices dont nous ne connaissons l'existence que par une inscription - le phénomène ne concerne que des théâtres et dont nous ne pouvons dire à quelle série ils appartiennent. C'est le cas des théâtres de Feurs (Loire) ou de Bram (Aude) ${ }^{63}$ ou encore des

58. F. Dumasy, Le théâtre d'Argentomagus (Saint-Marcel, Indre), p. 231.

59. Ibid. p. 231.

6o. Vendoeuvres-en-Brenne: CIL XIII 11151 ; Néris-les-Bains: CIL XIII 1376-1377 et $1379 \mathrm{~d}$.

6I. M. Dondin-Payre, «Sanctuaires publics et territoires civiques», p. 153.

62. F. Dumasy, «Petit atlas des édifices de spectacle en Gaule romaine», p. 48-52.

63. Feurs: CIL XIII 1642; Bram: M. Passelac, "Dédicace du théâtre de Bram (Aude)». 
édifices identifiés par le remploi de leurs gradins dans des fortifications tardives autour de Trèves. Il a fallu créer une catégorie indéterminée, car certains théâtres comme celui du sanctuaire de Lenus Mars à Trêves sont trop mal connus pour qu'on puisse les classer. Enfin, pour l'instant, nous avons installé les trois théâtres d'Avenches, d'Augst et de Mandeure dans une série spécifique qui présente tous les dispositifs habituels aux théâtres de tradition romaine, mais dont la scène, inscrite dans l'orchestra, relève du modèle gallo-romain.

Il est évident qu'une carte comme celle-ci suscite le débat car elle apparaît comme un instantané de nos connaissances: elle attend donc compléments ou corrections. Si nous la livrons ici, c'est pour faire apparaître les traits principaux de la carte du spectacle en Gaule. On constate que les colonies ou les chefs-lieux de Narbonnaise possèdent l'équipement nécessaire pour répondre aux prescriptions officielles: la majorité d'entre eux est dotée d'un amphithéâtre et d'un théâtre. C'est aussi le cas de quelques cités méridionales de l'Aquitaine, Agen, Cahors et Périgueux ${ }^{64}$.

En revanche, il saute aux yeux que la répartition spatiale des édifices de spectacle dans les Trois Gaules relève d'un schéma différent. On a depuis longtemps souligné la présence, habituelle au chef-lieu, de l'amphithéâtre et l'absence du théâtre, alors que se dressent, dans les agglomérations et les sanctuaires dispersés sur le territoire de la cité, des théâtres - tous de type gallo-romain. Ces édifices, équipés d'un gradin d'honneur, accueillent, à tour de rôle, des ludi scaenici en l'honneur de leurs dieux protecteurs et de ceux de la cité. Cette dispersion renvoie à ce qu'on pourrait décrire comme une politique des spectacles à l'échelle de la cité, avec une spécialisation des rôles: au chef-lieu, l'amphithéâtre, symbole de la richesse des élites et de la romanité, aux agglomérations et aux sanctuaires, le théâtre et ses ludi, associant divinités locales, dieux de la cité et culte impérial, comme l'indique l'inscription du théâtre d'Eu.

Ce «modèle" ne s'applique pas de façon uniforme et l'on repère dans telle ou telle cité des organisations différentes, liées aux contextes locaux et aux choix des élites. Citons quelques cas spectaculaires: Amiens, Autun, Limoges, Lutèce, Meaux, Trêves sont équipés et d'un

64. On note en revanche que certaines villes de Narbonnaise ne possèdent qu'un seul édifice: nous ne commenterons pas ici ces absences qui tiennent soit à l'état de la recherche, soit à l'histoire de chacun des sites. 
Fig. 1 - Théâtres et amphithéâtres en Gaule à la fin du II siècle. 
amphithéâtre et d'un théâtre auxquels s'ajoutent, dans trois de ces villes, un théâtre suburbain. Les élites de Tours ont fait le choix de n'installer aucun théâtre permanent sur leur territoire ${ }^{65}$ : le seul édifice voué aux spectacles est l'amphithéâtre. De bonnes dimensions lors de sa construction, il est agrandi au milieu du $\mathrm{II}^{\mathrm{e}}$ siècle et se hisse parmi les cinq amphithéâtres les plus grands de l'Empire ${ }^{66}$. Cette cité au territoire restreint s'affirme dans un choix centralisateur et monumental qui la distingue de ses voisines pictone, carnute et biturige.

Par ailleurs, on remarque que certaines cités sont dépourvues de tout édifice de spectacle; dans certains cas, ces absences sont dues aux insuffisances de la recherche: rappelons la découverte récente d'un théâtre à Javols, chef-lieu des Gabales, ou à Blicquy, l'un des grands sanctuaires de la cité des Nerviens. En revanche, il est moins sûr que le faible équipement des cités de la Lyonnaise de l'Ouest soit à imputer aux lacunes de l'activité archéologique. On constate qu'aucune n'élève un amphithéâtre ou un théâtre de tradition romaine; lorsqu'elles construisent un édifice de spectacle, elles optent en faveur d'un théâtre de type gallo-romain ou d'un "édifice à arène". Il y a là des choix différents de ceux faits par la majorité des cités: sont-ils dus à des ressources limitées? Ou à la volonté d'afficher des édifices de type gallo-romain?

Cette carte suscite en fait de nombreux commentaires que nous ne poursuivrons pas dans le cadre de cet article. Disons en conclusion qu'elle révèle à la fois des tendances générales, des spécificités régionales, des choix parfois très différents d'une cité à l'autre et à l'intérieur même des cités, des concurrences, voire des émulations qui aboutissent à des densités particulièrement élevées chez les Veliocasses ou les Bituriges ou à des vides remarquables comme chez les Turons.

65. Certains chercheurs, en particulier les membres du Projet Collectif de Recherches "Atlas de Touraine», se prononcent pour le rattachement du sanctuaire de Clion - inclus dans le territoire du diocèse de Berry - au territoire turon. La discussion reste ouverte.

66. J. Seigne, «Les trois temps de l'amphithéâtre antique». 


\section{Fonction ludique}

Nous nous intéresserons essentiellement aux ludi scaenici, en laissant de côté les munera et les venationes. En effet, plusieurs expositions et ouvrages récents ont exploré l'iconographie et l'épigraphie amphithéâtrale et enrichi notre connaissance des spectacles donnés dans l'arène ${ }^{67}$. Leur création à l'époque romaine, leur nature, leur déroulement selon des codes bien connus rendent leur histoire relativement simple. Il n'en va pas de même des ludi dont la tradition remonte aux histrions étrusques, qui bénéficient de tous les enrichissements artistiques de l'époque hellénistique, qui se nourrissent d'une riche tradition littéraire

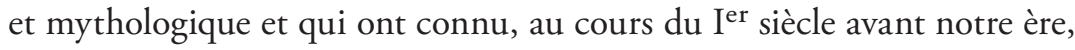
des évolutions complexes liées à la diffusion de la musique et de la danse sous des formes renouvelées ${ }^{68}$. Cette effervescence du milieu théâtral se traduit par la création à l'époque d'Auguste, en 23 ou en 22 avant notre ère, d'un nouveau spectacle, la pantomime. Un acteur unique, vêtu d'un costume luxueux, le visage couvert d'un masque à bouche fermée, exécute une danse muette, toute d'expressivité et de mimétisme. Il est accompagné d'un chœur qui chante sur un livret écrit par un auteur renommé et d'un orchestre de musiciens dont l'importance varie selon les ressources du commanditaire.

C'est à deux danseurs de renom, Bathylle, originaire d'Alexandrie et affranchi de Mécène et surtout Pylade, venu de Cilicie et affranchi d'Auguste, que l'on doit la création de ce nouveau genre dramatique. Comme le précise Marie-Hélène Garelli :

Pylade inventa la pantomime à grand spectacle [...] par association de pratiques diverses: il greffait sur une gestuelle dansée issue du mime qui existait déjà dans le monde gréco-romain, des innovations musicales $[. .$.$] et des arguments mythologiques que la popularité des$ tragédies d'Euripide avait contribué à répandre ${ }^{69}$.

67. Citons notamment depuis la parution de l'ouvrage de G. Ville, La gladiature en Occident des origines à la mort de Domitien, le colloque de Lattes édité par C. Domergue, Ch. Landes et J.-M. Pailler, Spectacula I et le catalogue édité par A. La Regina (ed.), Sangue e arena.

68. F. Dupont, L'Acteur-roi, p. 69-91.

69. M.-H. Garelli, Danser le mythe, p. 160. 
Ces innovations sont apparues au moment où Auguste s'intéressait au théâtre et à sa capacité à rassembler les foules. Le spectacle total que constituait une pantomime pouvait contribuer à un projet d'«unification culturelle» qui s'exprime à Rome et dans les provinces, par la construction de théâtres à la capacité d'accueil accrue et par les programmes décoratifs des frontes scaenarum. Elle n'a d'ailleurs pas chassé le mime à contenu mimétique, parodique et bouffon ni les diverses variantes de spectacle scénique qui s'épanouissent au long de la période impériale ${ }^{70}$.

On comprend mieux, dans ce contexte, le succès dont jouit le théâtre en Narbonnaise, puis dans les Trois Gaules. Et cependant, on souligne souvent l'absence en Gaule de témoignage sur les ludi scaenici euxmêmes. C'est oublier la documentation recueillie à Nîmes sur l'existence d'une association d'artistes dionysiaques, membre de «la sainte confrérie universelle des artistes au service de Dionysos et de l'Empereur", autour de laquelle devaient graviter des pantomimes et des musiciens ${ }^{71}$. Lui fait écho la stèle funéraire des scaenici Asiaticiani indiquant qu'à Vienne ce grand et riche personnage qu'est Valerius Asiaticus entretenait une troupe d'acteurs qu'il pouvait probablement louer à qui le lui demandait, selon une pratique bien attestée ${ }^{72}$. Un dernier document, daté de 198 de notre ère indique qu'à Bitburg, dans la cité trévire, Lucius Ammiatius Gamburio finance la construction d'un proscaenium cum tribunali. Avec les intérêts du capital engagé, il finance également l'entretien du proscaenium et l'édition de jeux scéniques annuels qui ont lieu la veille des calendes de mai $^{73}$. Il s'agit en fait des Ludi Florales qui sont célébrés à Rome du 28 avril au 3 mai et qui comportaient des circenses, des chasses et des ludi scaenici. On s'aperçoit que dans cette agglomération trévire, c'est une déesse romaine qui est honorée. Il n'est cependant pas exclu que le caractère licencieux des fêtes de Flore ait séduit l'évergète qui ne finance, en tout cas, que les ludi scaenici...

En dépit de ces informations, certains chercheurs ont contesté l'idée que les théâtres de type gallo-romain aient pu accueillir des ludi scaenici.

70. Comme en témoigne le spectacle donné au théâtre de Corinthe qu'Apulée nous décrit au livre $\mathrm{X}$ de son roman L'âne d'or et qui apparaît très difficile à classer.

7I. P. Ghiron-Bistagne, "Les concours grecs en Occident, notamment à Nîmes", p. 225-230 et M. L. Caldelli, "Gli agoni alla greca nelle regioni occidentali dell'Impero".

72. CIL XII 1929.

73. CIL XIII 4132. 
Frappés par les faibles dimensions de la scène, par son "exiguïté», ils lui refusent toute fonction théâtrale et considèrent qu'il s'agit d'une sorte d'estrade ou de tribune sur laquelle étaient placées les effigies impériales, ajoutant que «l'essentiel du jeu des acteurs, sinon sa totalité, devait se situer dans l'orchestra " ${ }^{74}$. Selon eux, ces édifices répondant à la fois à des «exigences liturgiques traditionnelles et récentes, celles du déroulement de spectacles étrangers à l'esprit gréco-romain et celles du culte impérial ${ }^{75}$, deviennent essentiellement des lieux de prière voués à l'empereur et à sa famille.

Or, toutes les «tribunes» citées présentent une superficie supérieure à $50 \mathrm{~m}^{2}$, ce qui permet à un acteur de pantomime ou de mime de se produire en solo. C'est également la superficie de la scène du petit théâtre de Pont-de-Metz (Somme): même dans une cavea de $30 \mathrm{~m}$ de diamètre, on a construit une estrade convenant aux évolutions d'un acteur $^{76}$. Il est d'ailleurs fréquent que les scènes soient agrandies, comme à Argentomagus où elle passe de 56 à $76 \mathrm{~m}^{2}$. Quant aux chanteurs et aux musiciens, ils pouvaient être accueillis dans l'orchestra qui complétait l'espace du jeu.

Autre trait qui confirme la vocation théâtrale des lieux, la présence d'une frons scaenae qui fait l'objet d'un décor peint ou sculpté et celle, à l'arrière de ce mur, d'un postscaenium qui se présente sous l'aspect d'une structure rectangulaire aux murs plus larges que ceux de la scène car ils portent une couverture. Le postscaenium est en effet un bâtiment fermé, adossé au mur rectiligne, dans lequel l'acteur se prépare avant de se présenter au public. Dans les théâtres romains, l'accès à la scène se fait à travers trois portes percées dans la frons scaenae. Au théâtre d'Argentomagus, la fouille a révélé la présence de deux portes placées aux extrémités de la frons $^{77}$. Ce passage de trois à deux tient probablement à la réduction de la longueur de la scène.

Il est essentiel, en fait, de rendre au théâtre sa fonction ludique: c'est parce que le théâtre est le lieu de jeux, de danses et de manifestations musicales donnés en l'honneur des dieux et de l'empereur que les élites,

74. M. Fincker, F. Tassaux, «Les grands sanctuaires "ruraux" d'Aquitaine et le culte impérial", p. 56.

75. P. Aupert, M. Fincker, F. Tassaux, «Agglomérations secondaires de l’Aquitaine atlantique», p. 59-61.

76. L. Blondiau, La Ferme aux Mouches, relais routier de Pont-de-Metz (Somme).

77. F. Dumasy, Le théâtre d'Argentomagus (Saint-Marcel, Indre), p. 202-212. 
prolongeant le projet impérial, l'ont diffusé sur le territoire des cités où il répondait à leur volonté de rassembler la population sur d'anciens lieux de culte - ou d'en créer de nouveaux. Contrairement, en effet, à ce qui est souvent affirmé, la scène n'a pas été supplantée par l'arène. Sans doute, au cours du $\mathrm{II}^{\mathrm{e}}$ siècle, voit-on augmenter le nombre des édifices où se donnent les munera et les venationes. Dans certaines cités, un ou deux édifices à arène, parfois un amphithéâtre, doublent l'édifice du chef$\operatorname{lieu}^{78}$. Il arrive même que, comme à Beaumont-sur-Oise, le théâtre soit transformé en édifice à arène, mais il garde son postscaenium et la possibilité de monter une scène en bois. C'est le cas également aux édifices de Drevant et de Sanxay qui assument une double fonction.

En fait, loin de se concurrencer, théâtres et amphithéâtres se complètent pour proposer au long du $\mathrm{II}^{\mathrm{e}}$ siècle une offre variée de spectacles. Cependant, la situation qu'illustre la carte proposée ici n'est atteinte qu'à l'époque sévérienne, après deux siècles d'équipement progressif qui n'exclut d'ailleurs pas des démolitions, des transformations ou des recompositions ${ }^{79}$. Et elle ne dure que quelques décennies: on sait en effet qu'à partir de 235 , sous l'effet des difficultés économiques et des guerres, les portes des édifices de spectacle se ferment, définitivement pour certains. D'autres, plus nombreux qu'on ne l'a longtemps pensé, s'ouvrent à nouveau, comme en témoignent des auteurs du IVe siècle et comme le confirment des données archéologiques qui révèlent des traces de réfection et d'utilisation ${ }^{80}$. Mais ceci est le dernier moment d'une histoire dont nous avons rapidement parcouru quelques-unes des premières pages.

Françoise Dumasy

Professeur émérite, Université Paris 1

78. F. Dumasy, «Les édifices de spectacle en Gaule du Nord», p. 460-463.

79. Parmi les théâtres démolis et non remplacés, citons ceux de Bliquy et de Trèves (Altbachtal).

8o. F. Dumasy, «Les édifices de spectacle dans le paysage urbain de la Gaule tardive». 


\section{BIBLIOGRAPHIE}

\section{Abréviation}

CIL Corpus inscriptionum latinarum consilio et auctoritate academiae litterarum regiae borussicae editum, Berlin, 1863-.

\section{Sources}

Tertullien, De Spectaculis, édition et traduction de Marie Turcan, Paris, Les Editions du Cerf, 1986.

Tite-Live, Histoire romaine, Livre VII, édition de Jean Bayet et traduction de Gaston Baillet, Paris, Les Belles Lettres, 1968.

\section{Etudes}

Aucher, Marie-Reine, Aucher, Maurice, «Le théâtre gallo-romain des Tours Mirandes à Vendeuvre-du-Poitou", Bulletin de la Société des Antiquaires de l'Ouest, 19 (1985), p. 573-584.

Aupert, Pierre, Fincker, Myriam, Tassaux, Francis, "Agglomérations secondaires de l'Aquitaine atlantique", in Villes et campagnes en Gaule romaine, éd. Pierre Gros, Paris, Editions du CTHS, 1998, p. 45-69.

Bayard, Didier, Massy, Jean-Luc, Amiens romain, Samarobriva Ambianorum, Amiens, Revue Archéologique de Picardie, 1983.

Blondiau, Lydie, La Ferme aux Mouches, relais routier de Pont-de-Metz (Somme), Document Final de Synthèse, SRA Picardie, 2007.

Brunaux, Jean-Louis, "Ribemont-sur-Ancre (Somme) : du trophée celtique au lieu de culte public gallo-romain", in Archéologie des sanctuaires en Gaule romaine, éd. William Van Andringa, Saint- 
Etienne, Publications de l'Université de Saint-Etienne, 2000, p. 133-156.

Busson, Didier, "Lutèce monumentale du $\mathrm{I}^{\mathrm{er}}$ au IV $\mathrm{IV}^{\mathrm{e}} \mathrm{s}$.", in Les villes romaines du Nord de la Gaule. Vingt ans de recherches nouvelles, éd. Roger Hanoune, Villeneuve d'Ascq, Revue du Nord, 2007, p. $257-270$.

Caldelli, Maria Letizia, "Gli agoni alla greca nelle regioni occidentali dell'Impero. La Gallia Narbonensis", Atti della Accademia nazionale dei Lincei, 394 (1997), p. 391-481.

De Michele, Pierre, "Découvertes récentes sur le théâtre antique d'Apt", Revue Archéologique de Narbonnaise, 36 (2003), p. 199-229.

Derks, Ton, "Le grand sanctuaire de Lenus Mars à Trèves et ses dédicaces privées: une réinterprétation", in Sanctuaires, pratiques cultuelles et territoires civiques dans l'Occident romain, éds Monique Dondin-Payre, Marie-Thérèse Raepsaet-Charlier, Bruxelles, Le Livre Timperman, 2006, p. 239-270.

Desbat, Armand, "Nouvelles recherches à l'emplacement du prétendu sanctuaire lyonnais de Cybèle: premiers résultats ", Gallia, 55 (1998), p. 237-278.

—, "La colonie de Plancus", in Lugdunum, naissance d'une capitale, éd. Armand Desbat, Gollion, Infolio, 2005, p. 63-68.

Domergue, Claude, Landes, Christian, Pailler, Jean-Marie (éds), Spectacula I. Gladiateurs et amphithéâtres. Actes du colloque tenu à Toulouse et à Lattes les 26, 27, 28 et 29 mai 1987, Lattes, Imago, 1990.

Dondin-Payre, Monique, "Sanctuaires publics et territoires civiques: réflexions à partir de l'exemple du Bois l'Abbé (cité des Ambiens)", in Sanctuaires, pratiques cultuelles et territoires civiques dans l'Occident romain, éds Monique Dondin-Payre, MarieThérèse Raepsaet-Charlier, Bruxelles, Le Livre Timperman, 2006, p. $135-158$.

Dufour, Gérard, «Le grand théâtre de Vendeuil-Caply (Oise). Essai de restitution du sacellum et de la thymélé», in Spectacula II. Le théâtre antique et ses spectacles. Actes du colloque tenu au musée archéologique Henri-Prades de Lattes les 27, 28, 29 et 30 avril 1989, éd. Christian Landes, Lattes, Imago, 1992, p. 103-112. 
Dumasy, Françoise, "Petit atlas des édifices de spectacle en Gaule romaine", in Le goût du théâtre à Rome et en Gaule romaine, éd. Christian Landes, Lattes, Imago, 1989 p. 43-75.

—, "Les édifices de spectacle», in Villes et agglomérations antiques $d u$ sud-ouest de la Gaule: histoire et archéologie. Actes du $2^{e}$ Colloque Aquitania, Bordeaux, 13-15 septembre 1990, dir. Louis Maurin, Bordeaux, Fédération Aquitania, 1992, p. 293-305.

-, Le théâtre d'Argentomagus (Saint-Marcel, Indre), Paris, Editions de la Maison des sciences de l'homme, 2000.

—, «Les édifices de spectacle en Gaule du Nord. De la typologie à la chronologie", in Les villes romaines du Nord de la Gaule. Vingt ans de recherches nouvelles, éd. Roger Hanoune, Villeneuve d'Ascq, Revue du Nord, 2007, p. 447-465.

—, "Les édifices de spectacle dans le paysage urbain de la Gaule tardive", in Les Jeux et les spectacles dans l'Empire romain tardif et dans les royaumes barbares, éds Emmanuel Soler, Françoise Thelamon, Rouen, Publications des Universités de Rouen et du Havre, 2008, p. 69-88 (Les cahiers du GRHis 19).

Dupont, Florence, L'acteur-roi, Paris, Les Belles Lettres, 1985.

Durand, Marc, "Un sanctuaire "rural": le temple gallo-romain de la forêt d'Halatte", in Archéologie des sanctuaires en Gaule romaine, éd. William Van Andringa, Saint-Etienne, Publications de l'Université de Saint-Etienne, 2000, p. 119-129.

Duval, Paul-Marie, "Théâtres et édifices sacrés", Revue des Etudes Anciennes, 59 (1957), p. 355-368.

Fincker, Myriam, TAssaux, Francis, «Les grands sanctuaires "ruraux" d'Aquitaine et le culte impérial", Mélanges de l'Ecole Française de Rome, Antiquité, 104 (1992), p. 41-76.

Formigé, Jules, Le théâtre romain de Vienne, Vienne, 1950.

Frézouls, Edmond, Les villes antiques de la France. Germanie Supérieure, 1, Besançon, Dijon, Langres, Mandeure, Strasbourg, AECR, 1988.

Garelli, Marie-Hélène, Danser le mythe. La pantomime et sa réception dans la culture antique, Louvain/Paris, Peeters, 2007.

Gayraud, Michel, Narbonne antique des origines à la fin du III siècle, 8 e Supplément à la revue Archéologique de Narbonnaise, 1981.

Ghiron-Bistagne, Paulette, "Les concours grecs en Occident, notamment à Nîmes", in Spectacula II. Le théâtre antique et ses spectacles. 
Actes du colloque tenu au musée archéologique Henri-Prades de Lattes les 27, 28, 29 et 30 avril 1989, éd. Christian Landes, Lattes, Imago, 1992, p. 223-232.

Gillet, Evelyne, Paridaens, Nicolas, Demarez, Léonce, «Le sanctuaire de Blicquy-"Ville d'Anderlecht" (prov. Hainaut, Belgique)", in Sanctuaires, pratiques cultuelles et territoires civiques dans l'Occident romain, éds Monique Dondin-Payre, MarieThérèse Raepsaet-Charlier, Bruxelles, Le Livre Timperman, 2006, p. 181-215.

Golvin, Jean-Claude, L'amphithéâtre romain. Essai sur la théorisation de sa forme et de ses fonctions, Talence, Université de Bordeaux III, 1988.

Gose, Erich, Der gallo-römische Tempelbezirk im Altbachtal zu Trier, Mainz am Rhein, P. von Zabern, 1972.

Grenier, Albert, Manuel d'archéologie gallo-romaine, III, 2: Ludi et circenses, Paris, Picard, 1958.

Gros, Pierre, "L'Augusteum de Nîmes", Revue Archéologique de Narbonnaise, 17 (1984), p. 123-134.

—, La Gaule Narbonnaise. De la conquête romaine au IIIe siècle apr. J.-C., Paris, Picard, 2008.

Hanson, John Arthur, Roman theater-temples, Princeton, University Press, 1959.

Holmgren, Jean, «Un sanctuaire gallo-romain avec théâtre à Clion-surIndre", Bulletin du Groupe d'Histoire et d'Archéologie de Buzançais, 12 (1980), p. 13-25.

Jullian, Camille, «Les arènes de Lutèce et les théâtres amphibies de la Gaule", Revue des Etudes Anciennes, 22 (1920), p. 187-203.

Kenyon, Katherine, "The Roman theater at Verulamium", Archaeologia, 84 (1934), p. 214-261.

Kolendo, Jerzy, «La répartition des places aux spectacles et la stratification sociale dans l'Empire romain", Ktéma, 6 (1981), p. 301-315.

Krier, Jean, "Le théâtre gallo-romain découvert en 1985 à Dalheim (Grand Duché de Luxembourg)", in Spectacula II. Le théâtre antique et ses spectacles. Actes du colloque tenu au musée archéologique Henri-Prades de Lattes les 27, 28, 29 et 30 avril 1989, éd. Christian Landes, Lattes, Imago, 1992, p. 121-132.

Kunnen, Peter-Hans, "Trèves-Augusta Treverorum", in La marque de Rome. Samarobriva et les villes du nord de la Gaule, dir. JeanLuc Collart, Jean-Olivier Guilhot, Roger Hanoune, Monique 
Dondin-Payre, catalogue d'exposition, Musée de Picardie, 2004, p. 63-72.

La Crorx, Camille de, Etude sur le théâtre gallo-romain des Bouchauds, Angoulême, 1908.

La Regina, Adriano (ed.), Sangue e arena, Milano, Electa, 2001.

Le Barrier, Christian, Ceyrat (Puy de Dôme), Puy de Montaudou. Rapport de fouille programmée, SRA Auvergne, 2005.

Le Bot-Helly, Anne, "Vienne du village gaulois à la capitale de cité (5e siècle av. J.-C. / $2^{\mathrm{e}}$ siècle apr. J.-C.)", in Les Allobroges, Gaulois et Romains du Rhône aux Alpes, catalogue d'exposition, dir. JeanPascal Jospin, Gollion, Infolio, 2002, p. 102-109.

Loustaud, Jean-Pierre, Limoges antique, Limoges, Association des antiquités historiques du Limousin, 2000.

Magnan, Danielle, "Les trois édifices de spectacle antiques de Meaux (Seine-et-Marne)» (à paraître).

Maniquet, Christophe, Le sanctuaire antique des Arènes de Tintignac, Limoges, Culture \& Patrimoine en Limousin, 2004.

Matter, Georg, Das römische Theater von Avenches/Aventicum. Architektur, Baugeschichte, kulturhistorische Aspekte, Lausanne, Cahiers d'archéologie romande, 2009 (Cahiers d'archéologie romande 114, Aventicum XV).

Naveau, Jacques, "Urbanisme et occupation du sol à Jublains", in Recherches sur Jublains (Mayenne) et sur la cité des Diablintes, éd. Jacques Naveau, Rennes, Documents Archéologiques de l'Ouest, 1997, p. 65-111.

Paillet, Jean-Louis, Petit, Catherine, «Nouvelles données sur l'urbanisme de Lugdunum des Convènes. Prospection aérienne et topographie urbaine», Aquitania, 10 (1992), p. 109-144.

Passelac, Michel, "Dédicace du théâtre de Bram (Aude). Vicus Eburomagus", in Le goût du théâtre à Rome et en Gaule romaine, éd. Christian Landes, Lattes, Imago, 1989, p. 229-230.

Rebourg, Alain, "L'urbanisme d'Augustodunum (Autun, Saône-etLoire)», Gallia, 55 (1998), p. 141-236.

Rivet, Lucien, Atlas topographique des villes de Gaule méridionale. 2, Fréjus, Montpellier, 32 Supplément à la Revue Archéologique de Narbonnaise, 2000.

Rossi, Frédéric (dir.), Théâtre d'Alésia. Rapport de synthèse 2004-2007, SRA Bourgogne, 2007. 
Roth-Congès, Anne, "L'acanthe dans le décor architectonique protoaugustéen en Provence», Revue archéologique de Narbonnaise, 16 (1983), p. 103-134.

Schwarz, Peter-Andrew, "Zur Chronologie und Typologie der drei Theaterbauten von Augusta Rauricorum ", Jahresberichte aus Augst und Kaiseraugst, 12 (1991), p. 33-96.

Seigne, Jacques, "Les trois temps de l'amphithéâtre antique», in Tours antique et médiéval. Lieux de vie, temps de la ville, éd. Henri Galinié, Tours, 30 $0^{\mathrm{e}}$ Supplément à la Revue Archéologique du Centre de la France, 2007, p. 238-246.

Thivet, Matthieu, Méthodes nouvelles de l'archéologie appliquées au site antique de Mandeure/Mathay (Doubs): reconnaissance spatiale, évolution chronologique, statut urbain, thèse de doctorat défendue à l'Université de Franche-Comté, 2008 (non publié).

Trillmich, William, "Un sacrarium del culto imperial en el teatro de Merida", Anas, 213 (1989-1990), p. 87-102.

Vermeersch, Didier, "L'agglomération antique de Beaumont-sur-Oise (Val d'Oise) : bilan des connaissances", in Les villes romaines $d u$ Nord de la Gaule. Vingt ans de recherches nouvelles, éd. Roger Hanoune, Villeneuve d'Ascq, Revue du Nord, 2007, p. 99-132.

Vermeersch, Didier, Wabont, Monique, "Genainville», in Le Vald'Oise, CAG 95, éds Monique Wabont, Franz Abert, Didier Vermeersch, Paris, Académie des Inscriptions et Belles Lettres, 2006, p. 270-277.

Vernou, Christian, La Charente, CAG 16, Paris, Académie des Inscriptions et Belles Lettres, 1993.

VIlle, Georges, La gladiature en Occident des origines à la mort de Domitien, Rome, Ecole française de Rome, 1981.

Wabont, Monique, Abert, Franz, Vermeersch, Didier, Le Val-d'Oise, CAG 95, Paris, Académie des Inscriptions et Belles Lettres, 2006. Wuilleumier, Pierre, Inscriptions latines des Trois Gaules, Paris, Editions du CNRS, 1984.

Crédits iconographiques

Fig. 1:

DAO Ludivine Lebrun. 